\title{
Open reduction internal fixation with triceps-sparing approach in distal humerus fracture: an experience from a tertiary level hospital in Mangalore
}

\author{
Bhaskar Bhandary, Sachin Shetty*, Mohammed Shabir Kassim, Amlan Mohapatra
}

Department of Orthopedics, AJ Institute of Medical Sciences, Mangalore, Karnataka, India

Received: 06 December 2018

Accepted: 12 January 2019

\author{
*Correspondence: \\ Dr. Sachin Shetty, \\ E-mail: departmentresearch2014@gmail.com
}

Copyright: (C) the author(s), publisher and licensee Medip Academy. This is an open-access article distributed under the terms of the Creative Commons Attribution Non-Commercial License, which permits unrestricted non-commercial use, distribution, and reproduction in any medium, provided the original work is properly cited.

\begin{abstract}
Background: Fractures of the distal humerus represent challenging problems to an orthopaedic surgeon. The present study aimed to assess the range of movement after performing open reduction and internal fixation of distal humerus fractures treated with triceps sparing approach.

Methods: This prospective study included all skeletally mature patients with distal humerus fractures and operated at our center with open reduction and internal fixation of distal humerus with triceps on or triceps sparing approach were included in the study. During the study period 30 cases underwent surgery and were included in the final analysis. Fractures were classified according to the AO/OTA classification. Patients will be followed up at 6 weeks, 12 weeks and at 6 months. Mean range of motion of the fractured elbow at different follow up points were compared.

Results: The mean age of the total population was $37.7 \pm 13.8$ years, $57 \%$ males and left side was affected in $60 \%$ of the patients. Majority of the patients had a range of motion in normal elbow in the range 0 to 140 degrees. There was an increase in the mean range of movement from $63.4 \pm 14.2$ at $6^{\text {th }}$ week to $120 \pm 6$ at $24^{\text {th }}$ week, and this change was statistically significant $(\mathrm{p}<0.001)$.

Conclusions: Future multicentric randomized studies, specially comparing triceps-sparing with olecranon osteotomy, are needed to support the results of our study.
\end{abstract}

Keywords: Intercondylar fractures of the distal humerus, Open reduction and internal fixation, Triceps-sparing

\section{INTRODUCTION}

Fractures of the distal humerus represent challenging problems to an orthopaedic surgeon. Historically, distal humeral fractures were treated nonoperatively due to the poor results associated with surgery. ${ }^{1}$ Literature, however, strongly supports open reduction and internal fixation of intra-articular distal humerus fractures. ${ }^{2}$ Surgery aims to restore anatomic structure of the articular surface and aligning joint with stable internal fixation, which would allow early range of motion. Olecranon osteotomy has been considered the gold standard technique for operating distal humerus fracture. It provides excellent exposure and avoids problems such as disruption of the extensor mechanism, fibrosis and intramuscular nerve injuries. ${ }^{3}$ However, osteotomies can be complicated with delayed union and can result in nonunion and prominent hardware. ${ }^{4}$ In 1982, Bryan and Morrey described an alternative technique of approaching the posterior elbow, in which the triceps mechanism was spared and reflected from medial to lateral without detachment. As the triceps-sparing approach uses relatively bloodless planes and avoids direct injury to the triceps muscle itself, scar formation is theoretically lower. This further helps to reduce elbow contracture postoperatively. By avoiding direct injury to the triceps, the risk of denervating a portion of the muscle is reduced, 
which may reduce the amount of postoperative triceps strength dysfunction. The present study aimed to assess the range of movement after performing open reduction and internal fixation of distal humerus fractures treated with triceps sparing approach.

\section{METHODS}

\section{Study design and sampling}

This prospective study included adults above the age of skeletal fusion, mostly above 21 years of age, presenting with distal humeral fractures, both intra-articular and extra-articular at AJ Institute of Medical Sciences, Mangalore from August 2015 to April 2017. All skeletally mature patients with distal humerus fractures and operated at our center with open reduction and internal fixation of distal humerus with triceps on or triceps sparing approach were included in the study. Patients with open injuries, associated with neurovascular injury, skeletally immature patients and pathological fractures. During the study period 30 cases underwent surgery and were included in the final analysis. The study was approved by the institutional ethics committee. All patients were explained the purpose of the study and an informed written consent was obtained from them.

\section{Surgical technique}

On admission of the patient a careful history was elicited from the patients and or attendants of injury and the severity of trauma. Radiograph of distal humerus i.e. antero-posterior view and lateral view were taken and fractures were classified according to AO/OTA classification. Patients were placed in the lateral position under suitable anaesthesia with the involved elbow at $90^{\circ}$ placed over an elbow support. General anaesthesia with aseptic precautions, open reduction and internal fixation of the fracture was done with plates of adequate size. The fascia overlying the triceps was then divided and two fasciocutaneous flaps were raised. The lateral and medial borders of the triceps were then readily visualized, as was the tricipital aponeurosis. The radial window was developed by initially lifting the lateral triceps from the lateral intermuscular septum and then the posterior surface of humerus. The ulna window was developed by lifting the medial triceps from the intermuscular septum and the dorsal surface of the humerus. In extra-articular distal humerus fractures, the two windows gave good enough access for fracture reduction, temporary fixation and application of $90 / 90$ or parallel plates. In simple intra-articular fractures, where no joint comminution was encountered, a combination of anatomical reduction of the condyles and intra-operative imaging of the joint surface was all that was needed for an accurate reduction and fixation. When the articular surface was comminuted, then the whole distal articular surface needed to be accessed. Connection of the medial and lateral dissections by mobilisation and elevation of the triceps muscle from the fracture and posterior humeral periosteum allowed visualisation of the entire posterior distal humerus. After that, the fracture was reduced and fixed provisionally with 1.5 or $2.0 \mathrm{~mm} \mathrm{~K}$ wires under fluoroscopic control in two planes. The intercondylar fracture was fixed with a 3.5-mm lag cortical screw in the coronal plane across the trochlea, thus converting the fracture into a supracondylar type. The articular fragment was then secured to the humeral shaft with two 3.5 reconstruction plates or condylar plates contoured to fit along the involved columns. An intraoperative radiograph was made to check reduction and fixation. The elbow was moved through a range of motion to test the stability of the fixation. The reconstructed distal articular block was then approximated to the humeral diaphysis. Kirschner-wire fixation was converted to definitive fixation with the application of either parallel or orthogonal plate constructs, depending on the surgeon's choice (Figure 1 and 2). All patients were admitted as in-patients and mobilization of the elbow was encouraged immediate postop.
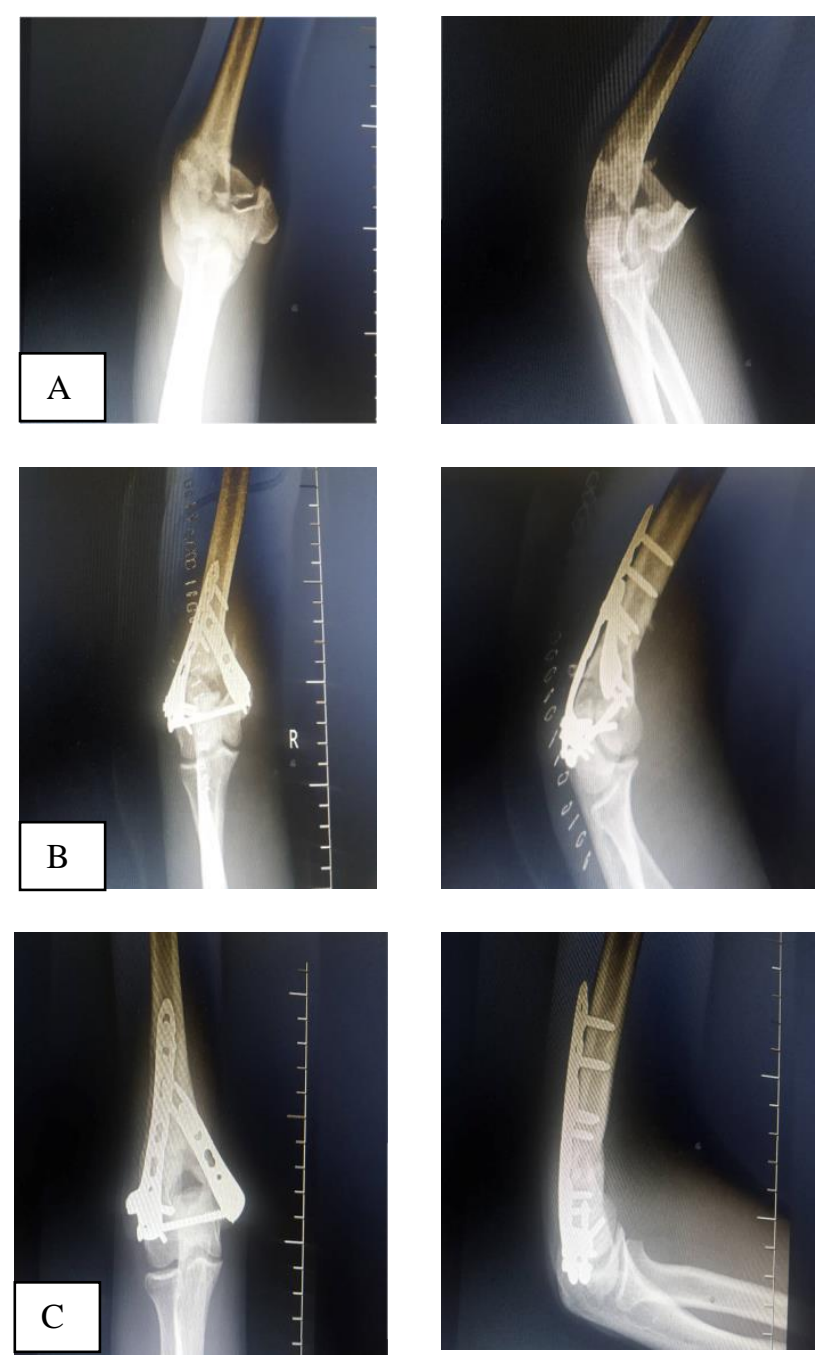

Figure 1: Radiographic assessment of a patient included in the study; (A) pre-operative assessment;

(B) immediate post-operative assessment; (C) at 6 weeks post-operative assessment. 

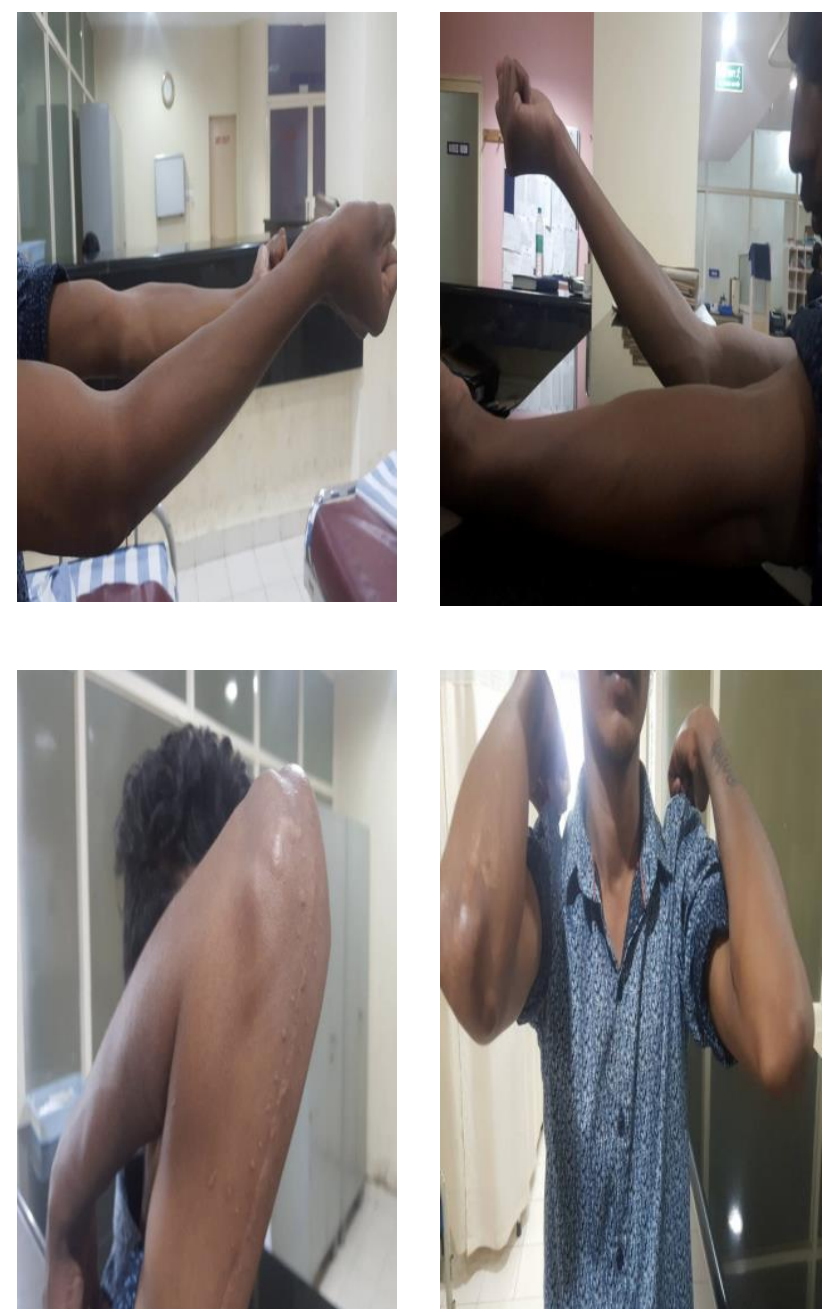

Figure 2: Clinical post-operative assessment of a patient included in the study.

\section{Data collection and data analysis}

Using a pre-tested semi-structured questionnaire, patient related clinical information was noted. Clinical and radiological evaluation was done. Fractures were classified according to the AO/OTA classification. Postoperative physiotherapy was followed according to the protocol, and functional outcome was assessed at 6 weeks, 12 weeks and at 6 months. All patients were encouraged to start range of motion exercises on the first day following surgery depending on the patient compliance and surgeon's discretion regarding a stable fixation. The unaffected elbow serves as a control for each patient. Anteroposterior and lateral elbow radiographs were obtained at the time of examination and assessed for reduction, alignment, fracture union, posttraumatic arthrosis, and heterotopic ossification. All data were analysed in SPSS version 21 (IBM Corp, NY). Quantitative and qualitative variables were described as mean (standard deviation) and frequency (percentage) respectively. Mean range of motion of the fractured elbow at different follow up points were compared using the Friedman test. A p value of less than 0.05 suggested statistical significance.

\section{RESULTS}

Table 1 describes the baseline demographic and clinical characteristics of the patients included in the study. It was found that $70 \%$ of the patients were below the age of 40 years. The mean age of the total population was $(37.7 \pm 13.8$ years), mean age among males (34.6 \pm 9.9 years) was lower as compared to that of females $(41.7 \pm 17.2$ years). There were $57 \%$ males and $43 \%$ females. Left side was affected in $60 \%$ of the patients. Patients were classified according to AO classification. There was only one case of extra-articular (A2moderate) type fracture. Partial articular fracture was seen in eight patients, of which one was simple, three were moderate and four were severe type. Intra-articular fracture was seen in 21 patients, of which eight patients had simple type, seven had moderate types and six patients were severe type. Majority of the patients had a range of motion in normal elbow in the range 0 to 140 degrees (Table 2). At 6 weeks the mean range of motion among the patients is $65.4 \pm 14.2$ degrees; at 12 weeks the mean range of motion among the patients is $98.5 \pm 9.1$ degrees; at 24 weeks the mean range of motion among the patients is $120.7 \pm 6.0$ degrees. There was an increase in the mean range of movement from $63.4 \pm 14.2$ at $6^{\text {th }}$ week to $120 \pm 6$ at $24^{\text {th }}$ week, and this change was statistically significant with a $\mathrm{p}<0.001$ (Figure 3).

Table 1: Baseline characteristics of the patients included in the study.

\begin{tabular}{|l|l|}
\hline Variables & N $(\%)$ \\
\hline Age distribution (years) & $12(40)$ \\
\hline 21 to 30 & $09(30)$ \\
\hline 31 to 40 & $02(07)$ \\
\hline 41 to 50 & $05(17)$ \\
\hline 51 to 60 & $02(06)$ \\
\hline More than 60 & \\
\hline Gender distribution & $13(43)$ \\
\hline Females & $17(57)$ \\
\hline Males & \\
\hline Affected side & $18(60)$ \\
\hline Left & $12(40)$ \\
\hline Right & \\
\hline Type of fracture & $01(03)$ \\
\hline Extra articular (A2-moderate) & \\
\hline Partial articular & $01(03)$ \\
\hline Simple (B1) & $03(10)$ \\
\hline Moderate (B2) & $04(13)$ \\
\hline Severe (B3) & \\
\hline Intra-articular & $08(27)$ \\
\hline Simple (C1) & $07(23)$ \\
\hline Moderate (C2) & $06(20)$ \\
\hline Severe (C3)
\end{tabular}


Table 2: Range of motion.

\begin{tabular}{|ll|}
\hline Range of motion in normal elbow \\
\hline 0 to 130 & $03(10)$ \\
\hline 0 to 140 & $14(47)$ \\
\hline 0 to 145 & $13(43)$ \\
\hline $\begin{array}{l}\text { Mean range of motion in fractured } \\
\text { elbow }\end{array}$ \\
\hline 6 weeks & $65.4 \pm 14.2$ \\
\hline 12 weeks & $98.5 \pm 9.1$ \\
\hline 24 weeks & $120.7 \pm 6.0$ \\
\hline
\end{tabular}

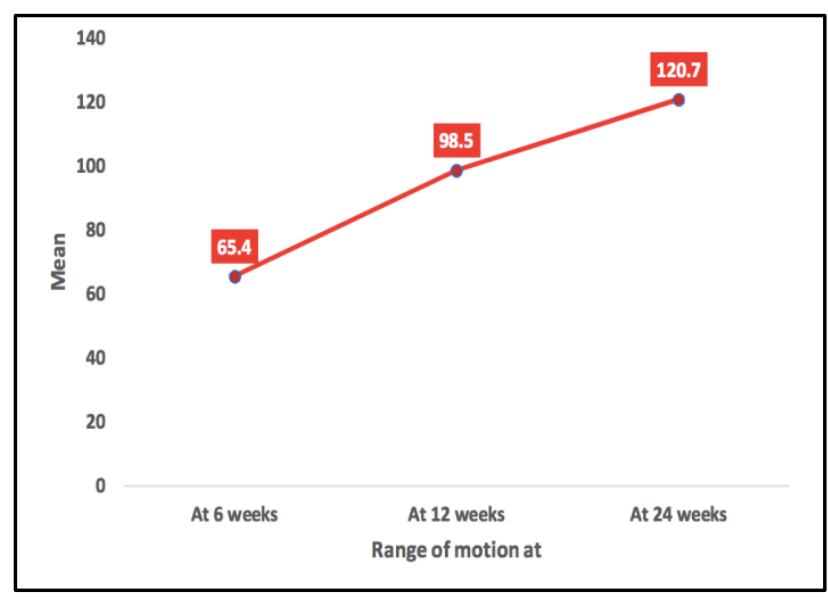

Figure 3: Range of motion on the fractured elbow $(n=30)$ at different follow up points.

\section{DISCUSSION}

The current study involves, evaluating the functional outcome and range of movements of distal humerus fractures treated with the triceps sparing approach. The triceps sparing approach has the advantage of not disrupting the extensor mechanism of the elbow, thereby allowing early mobilisation and preventing elbow stiffness. There are adequate number of studies that has evaluated the triceps sparing approach for the distal humerus and a majority of the studies give good follow up results. The triceps-sparing approach was originally described by Bryan and Morrey for its use in total elbow arthroplasty. ${ }^{5}$ They reported that, compared to other approaches, there was no loss of triceps function or significant weakness, and it allowed patients early active elbow motion and rehabilitation because the extensor mechanism is not disrupted in any way. This has also been demonstrated by other authors that by using the Bryan-Morrey technique for the treatment of intercondylar distal humeral fractures in adolescents, no significant loss in triceps strength resulted. In the present study, no patient reported triceps weakness on physical examination, although an accurate and objective musclestrength testing was not done.

The interpretation of overall functional outcome of distal humeral fractures from previous studies is difficult, as the populations studied are small and operate upon by multiple surgeons via multiple approaches. Varied rehabilitation protocols and inconsistent functional outcome measure scales make the comparison even more difficult. We observed a significant improvement in the range of motion post-operatively. Mean range of motion improved from $63.4 \pm 14.2$ at $6^{\text {th }}$ week to $120 \pm 6$ at $24^{\text {th }}$ week. Morrey et al concluded that $100^{\circ}$ of elbow flexion and $100^{\circ}$ of forearm rotation are required for most of the activities of daily living. ${ }^{6}$ Furthermore, on comparing triceps sparing and triceps splitting approaches for extraarticular distal humerus fractures, elbow range of motion and triceps strength were found to be better with a triceps sparing. ${ }^{7}$

There are a few limitations of this study. First, we had a small sample size and from a single centre. So the results of this study might not be applicable to other surgical centres and will vary with the surgical expertise and postoperative rehabilitation regime. Second, out study lacked any control groups for comparison. Finally, objective muscle strength testing of triceps could not be done.

\section{CONCLUSION}

Open reduction and internal fixation with triceps-sparing approach resulted in a significant improvement in the range of motion during subsequent post-operative follow ups. Future multicentric randomized studies, specially comparing triceps-sparing with olecranon osteotomy, are needed to support the results of our study.

\section{Funding: No funding sources}

Conflict of interest: None declared

Ethical approval: The study was approved by the institutional ethics committee

\section{REFERENCES}

1. Riseborough EJ, Radin EL. Intercondylar $\mathrm{T}$ fractures of the humerus in the adult. A comparison of operative and non-operative treatment in twentynine cases. J Bone Joint Surg Am. 1969;51:130-41.

2. Caja VL, Moroni A, Vendemia V, Sábato C, Zinghi G. Surgical treatment of bicondylar fractures of the distal humerus. Injury. 1994;25:433-8.

3. Gofton WT, Macdermid JC, Patterson SD, Faber KJ, King GJ. Functional outcome of AO Type C distal humeral fractures. J Hand Surg. 2003;28:294308.

4. McKee MD, Wilson TL, Winston L, Schemitsch EH, Richards RR. Functional outcome following surgical treatment of intra-articular distal humeral fractures through a posterior ap- proach. J Bone Joint Surg Am. 2000;82(A):1701-7.

5. Bryan RS, Morrey BF. Extensive posterior exposure of the elbow. A triceps-sparing approach. Clin Orthop Relat Res. 1982;166:188-92.

6. Morrey BF, Askew LJ, Chao EY. A biomechanical study of normal functional elbow motion. The 
Journal of bone and joint surgery. American volume. 1981;63(6):872-7.

7. Illical EM, Farrell DJ, Siska PA, Evans AR, Gruen GS, Tarkin IS. Comparison of outcomes after triceps split versus sparing surgery for extraarticular distal humerus fractures. Injury. 2014;45(10):1545-8.
Cite this article as: Bhandary B, Shetty S, Kassim MS, Mohapatra A. Open reduction internal fixation with triceps-sparing approach in distal humerus fracture: an experience from a tertiary level hospital in Mangalore. Int J Res Orthop 2019;5:345-9. 